\title{
Repeat surgery for focal cortical dysplasias in children: indications and outcomes
}

\author{
Matthew F. Sacino, BS, ${ }^{1}$ Cheng-Ying Ho, MD, PhD, ${ }^{3}$ Matthew T. Whitehead, MD, ${ }^{2}$ Amy Kao, MD, ${ }^{4}$ \\ Dewi Depositario-Cabacar, MD, ${ }^{4}$ John S. Myseros, MD, ${ }^{1}$ Suresh N. Magge, MD, ${ }^{1}$ \\ Robert F. Keating, MD, ${ }^{1}$ William D. Gaillard, MD, ${ }^{4}$ and Chima O. Oluigbo, MD${ }^{1}$
}

\begin{abstract}
Departments of ${ }^{1}$ Neurosurgery, ${ }^{2}$ Neuroradiology, ${ }^{3}$ Neuropathology, and ${ }^{4}$ Neurology, Children's National Health System, Washington, DC
\end{abstract}

\begin{abstract}
OBJECTIVE Focal cortical dysplasia (FCD) is a common cause of medically intractable epilepsy that often may be treated by surgery. Following resection, many patients continue to experience seizures, necessitating a decision for further surgery to achieve the desired seizure outcomes. Few studies exist on the efficacy of reoperation for intractable epilepsy due to FCD in pediatric cohorts, including the definition of prognostic factors correlated with clinical benefit from further resection.
\end{abstract}

METHODS The authors retrospectively analyzed the medical records and MR images of 22 consecutive pediatric patients who underwent repeat FCD resection after unsuccessful first surgery at the Children's National Health System between March 2005 and April 2015.

RESULTS Accounting for all reoperations, 13 (59\%) of the 22 patients achieved complete seizure freedom and another 5 patients $(23 \%)$ achieved significant improvement in seizure control. Univariate analysis demonstrated that concordance in electrocorticography $(E C O G)$ and MRI localization $(p=0.005)$, and completeness of resection $(p=0.0001)$, were associated with seizure freedom after the first reoperation. Patients with discordant ECoG and MRI findings ultimately benefited from aggressive multilobe lobectomy or hemispherectomy. Repeat lesionectomies utilizing intraoperative MRI (iMRI; $n=9$ ) achieved complete resection and seizure freedom in all cases.

CONCLUSIONS Reoperation may be clinically beneficial in patients with intractable epilepsy due to FCD. Patients with concordant intraoperative ECoG and MRI localization may benefit from extended resection of residual dysplasia at the margins of the previous lesional cavity, and iMRI may offer benefits as a quality control mechanism to ensure that a complete resection has been accomplished. Patients with discordant findings may benefit from more aggressive resections at earlier stages to achieve better seizure control and ensure functional plasticity.

https://thejns.org/doi/abs/10.3171/2016.8.PEDS16149

KEY WORDS reoperation; focal cortical dysplasia; intraoperative MRI; epilepsy surgery

$\mathrm{F}$ OCAL cortical dysplasia (FCD) is a common cause of intractable epilepsy in children and often requires surgical intervention. Following first surgery, 33\%$54 \%$ of patients continue to have seizures. ${ }^{1,3,6,7,10,14,20}$ In such cases, a decision must be made on whether to undertake further resection, and to what extent the resection in the reoperation should be tailored (i.e., lesionectomy, lobectomy, or hemispherectomy).

Few studies exist on reoperation for intractable epilepsy in pediatric populations. Two of the largest cohorts have reported on the clinical benefit of reoperation, with $55 \%-$ $61 \%$ seizure freedom after $1-3$ reoperations. ${ }^{16,19}$ A vital component of the decision to reoperate is the identification of patients who are more likely to achieve clinical benefit from additional resection. Prognostic factors correlated with favorable seizure outcome following reoperation include shorter duration of epilepsy prior to first surgery, focal discharges on the preoperative interictal electroencephalography (EEG), and concordance of ictal EEG findings prior to reoperation with ictal EEG and MRI findings

ABBREVIATIONS ECOG = electrocorticography; EEG = electroencephalography; FCD = focal cortical dysplasia; iMRI = intraoperative MRI; vEEG = video EEG; VNS = vagus nerve stimulator. SUBMITTED March 11, 2016. ACCEPTED August 25, 2016.

INCLUDE WHEN CITING Published online November 11, 2016; DOI: 10.3171/2016.8.PEDS16149. 
prior to first surgery. ${ }^{9,20}$ Surgical factors associated with clinically beneficial reoperations include repeat resection of residual dysplasia in patients with focal lesions who failed first surgery due to incomplete resections, and multilobar or hemispheric resections in patients with extensive epileptogenic zones or dysplasia not clearly indicated on imaging. ${ }^{2,16,20,26}$ Given that reoperations increase the risk of complications and morbidity, there is a need to develop refined clinical and diagnostic criteria to determine which patients are more likely to benefit from reoperation. The objective of this study was to examine whether further repeat resections result in improved seizure control in pediatric patients with intractable epilepsy secondary to FCD who did not achieve desired clinical outcomes after first surgery.

\section{Methods}

\section{Patient Population}

Between March 2005 and April 2015, 22 pediatric patients underwent reoperation for intractable epilepsy secondary to FCD or heterotopia. The inclusion criteria for this study were: 1) repeat resection for medically intractable epilepsy at the Children's National Health System and 2) preoperative clinical diagnosis and postoperative histopathological confirmation of FCD or heterotopia after first surgery. The decision to offer reoperation at our institution is made by a multidisciplinary epilepsy team. The current study was approved by the Institutional Review Board of the Children's National Health System.

\section{Reoperations}

Before each subsequent repeat surgery, all patients underwent brain MRI using our dedicated epilepsy imaging protocol on a 1.5- or 3.0-T MRI machine (Greenline Achieva Nova Dual, Philips Medical System) to document the extent of residual dysplasia. Patients also underwent prolonged video EEG (vEEG) for seizure lateralization and localization. Wada testing or functional MRI with motor and language paradigms was performed in patients with foci in peri-eloquent cortex. Implantation of subdural grids and electrodes and extraoperative monitoring were performed in 7 patients. The decision to perform extraoperative monitoring was made prior to surgery by the multidisciplinary epilepsy team.

In cases in which MRI suggested residual dysplasia after the first surgery, the surgical plan of reoperation was an extended resection at the margins of the lesion cavity. We previously described the conventional microsurgical technique for resection of FCD at our institution. ${ }^{13}$ Intraoperative MRI (iMRI) was incorporated into the final 9 cases of this cohort as a quality control technique that allowed for the intraoperative confirmation of completeness of resection of the MRI lesion. In cases of discordant EEG spikes more than $10 \mathrm{~mm}$ from the MRI-documented lesion, resection was primarily aimed at resecting abnormal cortex indicated by intracranial electrodes, with the thought that the epileptic zone extended beyond predominant FCD foci. More aggressive resection, including multilobe lobectomy and hemispherectomy, was performed in 3 cases.

Postoperative seizure outcomes were assessed at the last postoperative follow-up evaluation using the Engel classification system. ${ }^{5}$ We assessed the correlation of seizure outcome with the following factors: duration of seizures, age at first surgery, age at second surgery, time to recurrence of seizures after first surgery, concordance of MRI and electrocorticography (ECoG) findings, and completeness of resection.

\section{Concordance}

Intraoperative coordinates of the EEG electrodes were based on 2 factors: 1) visual assessment of the relationship of the electrode to the gyrus that had been determined to contain the cortical dysplasia, based on intraoperative neuronavigation, and 2) visual assessment of local anatomy. In cases of cortically implanted electrodes, volumetric postoperative MR images were obtained that confirmed the location of the electrodes in relationship to the gyrus that contained the FCD.

We reviewed the preoperative MRI, intraoperative ECoG, invasive subdural monitoring, and surgical records for all cases. Concordance was analyzed by comparing the spatial relationship of the lesion-as localized by neuronavigation-based preoperative MRI findings - to intracranial EEG findings. Interictal spikes localized to the MRindicated lesional cavity were categorized as concordant. Spikes recorded more than $10 \mathrm{~mm}$ or 1 gyrus distal to the MR-indicated lesional cavity were categorized as discordant. $^{24}$

\section{Statistical Analysis}

Clinical and demographic statistics were recorded as means \pm standard deviations, and duration of follow-up was reported as median number of months. Statistical analysis of continuous variables between the independent cohorts was performed via the nonparametric Mann-Whitney U-test. The Fisher exact test was used to assess categorical variables between groups. The Wilcoxon rank-sum test was performed among univariate variables. All tests performed were 2 -tailed, and a $\mathrm{p}$ value $<0.05$ was considered statistically significant.

\section{Results \\ Patient Demographics}

Twenty-two patients (14 boys, 8 girls) met the inclusion criteria. The duration of seizures prior to first surgery ranged from 1 to 150 months (mean $48.6 \pm 45.2$ months), age at first surgery ranged from 3 months to 17 years (mean $5.8 \pm 4.4$ years), and age at second surgery ranged from 8 months to 19 years (mean $7.1 \pm 5.0$ years).

Clinical and surgical characteristics are summarized in Table 1. Seventeen patients (77\%) presented with focal seizures only and 4 (18\%) of the patients (Cases 3, 7, 9 , and 11) presented with focal and generalized seizures. Two patients (Cases 7 and 11) initially presented with epileptic encephalopathy and 1 patient (Case 13) presented with infantile spasms associated with Aicardi's syndrome. Fourteen patients $(64 \%)$ presented with FCD or heterotopia in peri-eloquent cortex. Ultimately, 7 patients (32\%) underwent a second reoperation and 1 patient underwent a third reoperation. 


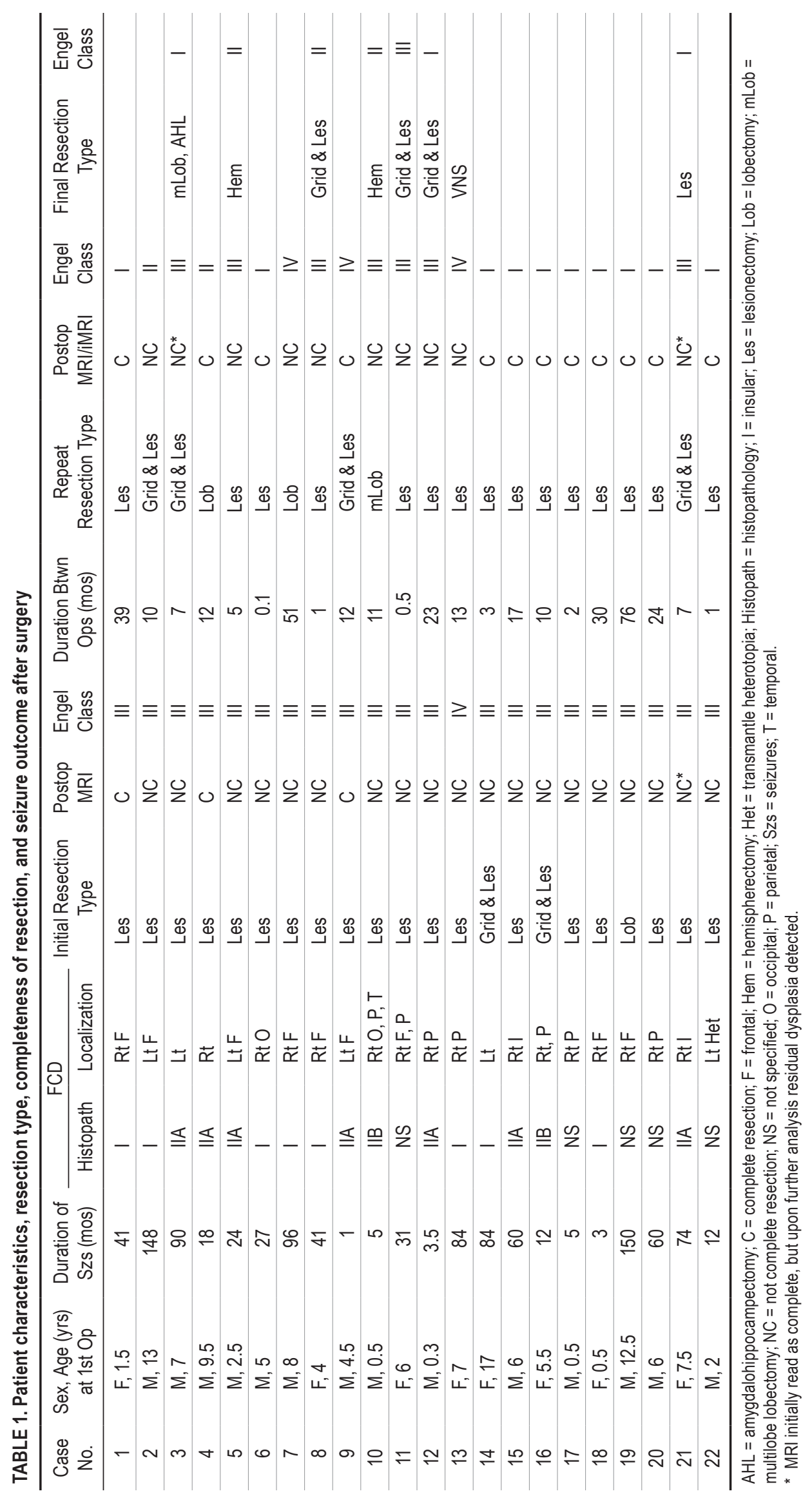




\section{First Surgery and Postoperative Results}

Following preoperative evaluation and review by the multidisciplinary epilepsy team, all patients underwent first surgery. Prior to surgery, 2 patients underwent extraoperative monitoring with grid electrodes. Twenty-one patients underwent a lesionectomy and 1 patient underwent a lobectomy (Table 1).

Postoperative MRI obtained within 24 hours of first surgery indicated residual FCD or heterotopia at the margins of the resection cavity in 18 of the 22 patients. Figure 1 illustrates residual FCD Type I (Case 6) and FCD Type IIa (Case 12) dysplasia after first surgery. Functional consideration of FCD localized to peri-eloquent cortex was the most prevalent cause of incomplete resection. The findings of the imaging completed in Cases 1, 4, 9, and 21 did not show any evidence of residual dysplasia. Twentyone of 22 patients experienced minor reduction in seizures following the first surgery (Engel Class III), while 1 patient had no change (Engel Class IV). The median duration to recurrence of seizures was 1.46 months (range 1 day to 20.6 months).

Histopathological specimens taken from the first surgery confirmed Type 1 FCD in 8 patients (36\%), Type 2A FCD in 7 patients (32\%), Type 2B FCD in 2 patients (9\%), and FCD of undetermined classification in 5 patients $(23 \%)$.

\section{Second Surgery: Preoperative Evaluation, Surgical Characteristics, and Results}

All patients underwent preoperative 1.5- $(\mathrm{n}=10)$ or 3.0$\mathrm{T}(\mathrm{n}=12)$ MRI and vEEG prior to the second surgery. MRI of lesional abnormalities and vEEG were concordant in each case except Cases 1, 4, 9, and 21, where dysplastic cortex could not be visualized on imaging prior to the second surgery despite EEG discharges arising from the cortical location of the first surgery. Intraoperative ECoG was concordant with both the ECoG findings from the first surgery or preoperative MRI in 15 patients (68\%). At surgery, 7 of the patients (32\%) had discordant ECoG findings, meaning that the spatial location of the abnormal ECoG findings was distant (>10 mm) from the lesion, as localized by neuronavigation-based preoperative focal MRI findings. Of these patients, 3 presented with global delay due to diffuse neurological pathology (Cases 7, 11, and 13), and 3 (Cases 3,5, and 10) initially presented with large diffuse lesions that spanned into other lobes. One patient (Case 9) who did not have residual dysplasia on initial postoperative MRI had ECoG findings of spikes in the cortex $2.5 \mathrm{~cm}$ distal from those recorded in previous ECoG.

Twelve (55\%) of the reoperations were a further resection of the most obvious MRI-indicated residual dysplasia at the margins of the previous resection cavity. Extraoperative invasive monitoring was contraindicated in Case 1 (hyperactive behavior) and Case 4 (autism spectrum disorder); instead, a resection of 2 gyri circumferentially around the preexisting cavity and temporal lobectomy was performed, respectively. Extraoperative invasive monitoring with subdural strips and grid electrodes was used in Case 2 for mapping of a seizure focus in close proximity to eloquent (language) cortex, in Case 3 to confirm
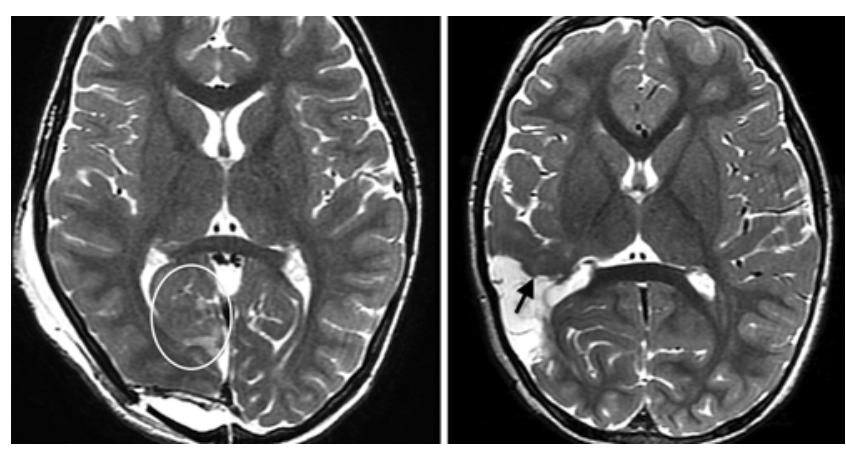

FIG. 1. Axial T2-weighted MR images. Left: Image illustrating residual FCD Type I cortical dysplasia (circled) in Case 6 following the first surgery. Right: Image illustrating residual FCD Type lla cortical dysplasia (arrow) in Case 12 following the first surgery.

concordance with MRI prior to the second surgery, and in Cases 9 and 21 to indicate concordance with MRI and ECoG recordings performed at the first surgery. Six patients (Cases 3, 5, 7, 10,11, and 13) underwent both an extended resection at the margins of the previous resection cavity of the MRI-indicated residual, as well as additional resection of secondary ECoG and/or MR-indicated foci, with 2 patients (Cases 7 and 10) undergoing lobectomies.

Intraoperative 1.5-T MRI $(\mathrm{n}=9)$ or postoperative 1.5$(n=9)$ or 3.0-T MRI $(n=4)$ confirmed complete resection in 9 of the 12 cases of extended resection of the MRI-indicated residual dysplasia. Of the 2 patients with postoperative MRI indicative of residual dysplasia who underwent extraoperative subdural and grid electrode monitoring, neither achieved a complete resection. Due to poor seizure control following the second operation, the patient in Case 21 underwent extensive reevaluation of postoperative scans and it was believed that there was suspicious dysplasia anterior to the resection cavity that was resected in a third operation. None of the 6 patients undergoing a second surgery of diffuse residual FCD achieved a complete resection after the second operation. Due to poor seizure control, 4 of these patients went on to have further operations. Patients 3, 5, and 10 later had a third surgery involving aggressive multilobe lobectomies or hemispherectomies as final operations, achieving a complete resection of FCD tissue in all cases. Patients 8, 11, and 12, who continued to have seizures despite resection of the most obvious MRI-indicated residual dysplasia at the second surgery, underwent a third surgery guided by extraoperative grid electrodes, with complete resection achieved in 1 $(33 \%)$ of 3 cases. Due to underlying Aicardi's syndrome, it was concluded that the patient in Case 13 was not a good candidate for further surgery and this patient underwent vagus nerve stimulator (VNS) implantation.

\section{Seizure Freedom Following Reoperation}

The median duration of postoperative follow-up after final resection was 13.56 months. Complete seizure freedom (Engel Class I) was achieved in 10 patients after a first reoperation, 2 after a second reoperation, and 1 after a third reoperation. Accounting for all reoperations, 13 patients $(59 \%)$ achieved complete seizure freedom (Engel Class I), 5 patients (23\%) continued to have rare disabling 


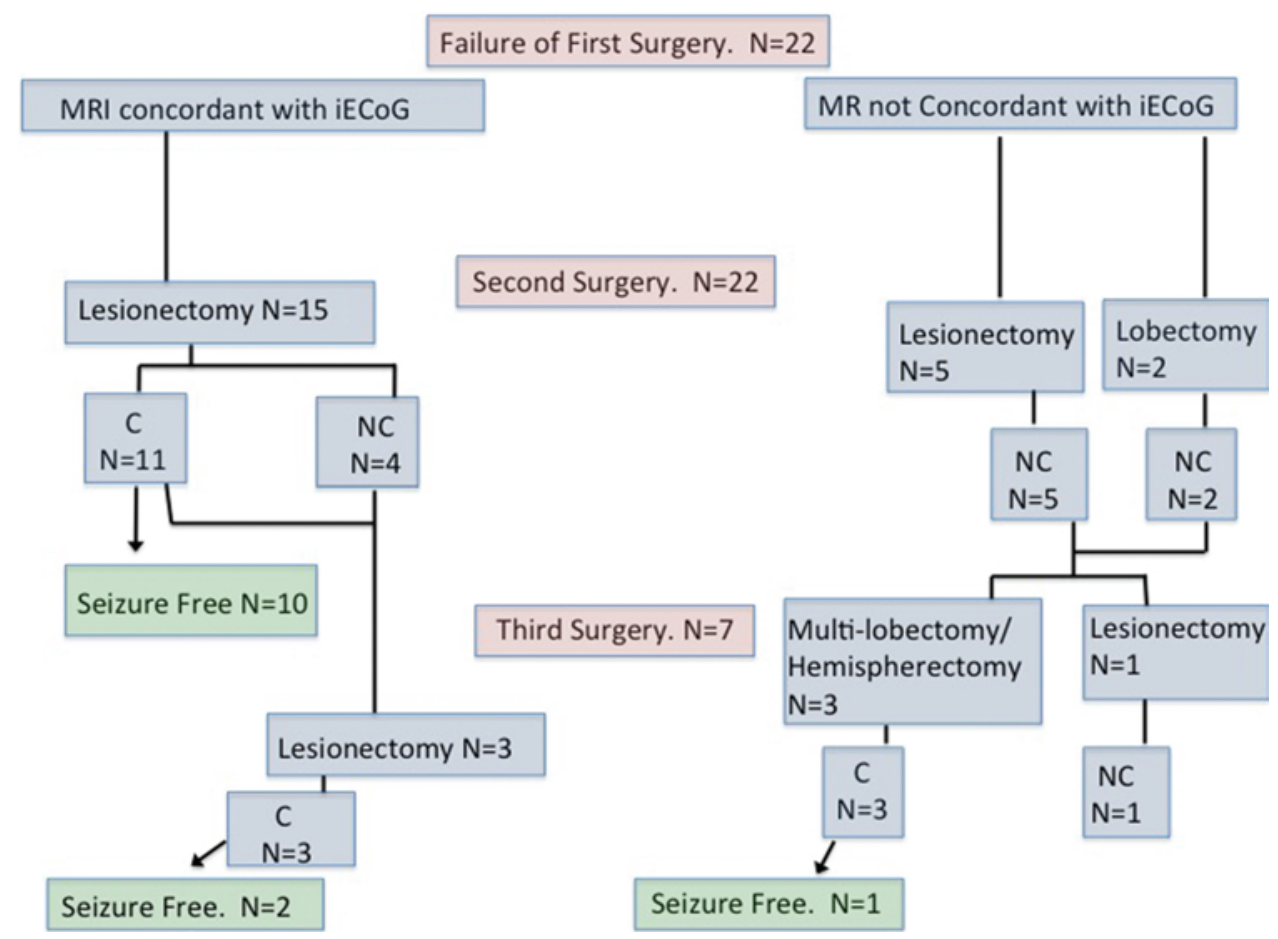

FIG. 2. Flow chart representing seizure outcomes based on diagnostic findings and resection type. $\mathrm{C}=$ complete resection; iECoG = intraoperative ECoG; NC = not complete resection. Figure is available in color online only.

seizures (Engel Class II), 1 patient (5\%) experienced only minor improvements in seizure frequency and severity (Engel Class III), and 3 patients (14\%) had no improvement (Engel Class IV).

\section{Localization of ECoG Findings and Seizure Control Outcomes}

Figure 2 summarizes seizure outcomes based on diagnostic findings and resection type. Following the second surgery, 10 (67\%) of the 15 patients with ECoG findings localized to the MRI-indicated lesion achieved complete seizure freedom. Two patients (Cases 2 and 4) achieved favorable seizure outcome (Engel Class II) following first repeat surgery. Patients 12 and 21 achieved complete seizure freedom after a third surgery, and Patient 8 achieved favorable seizure outcome (Engel Class II) after a third surgery.

None of the patients with discordant findings achieved complete seizure freedom following the second surgery. Three patients achieved favorable seizure outcome (Engel Class I and II) after third (Cases 5 and 10) and fourth (Case 3) surgeries. The patient in Case 11 had only minor reduction in seizures following third surgery. The patient in case 13 had no reduction in seizure frequency following VNS implantation on third surgery. The patient in Case 3 ultimately underwent a multilobar resection on the fourth surgery to achieve complete seizure freedom (Engel Class I). The patients in Cases 5 and 10 underwent hemispherectomy on the third operation, achieving favorable seizure outcome (Engel Class II).

Univariate analysis (Table 2) showed that the only significant predictors of seizure freedom following second surgery were: 1) concordance in ECoG and MRI localization of dysplastic foci (10 of 15 patients [67\%] with concordant findings vs 0 of 7 [0\%] with discordant findings; $\mathrm{p}=$ $0.005)$; and 2) complete resection of the MR-indicated foci

TABLE 2. Univariate analysis of predictive factors of surgical outcome

\begin{tabular}{lccc}
\hline \multicolumn{1}{c}{ Predictive Factor } & Engel Class I (\%) & Engel Class II-IV (\%) & $p$ Value \\
\hline Mean duration of Szs \pm SD (mos) & $45.4 \pm 14.5$ & $51.3 \pm 13.4$ & 0.79486 \\
\hline Mean age at 1st op \pm SD (yrs) & $5.6 \pm 1.7$ & $5.9 \pm 1.1$ & 0.62414 \\
\hline Mean age at 2nd op \pm SD (yrs) & $7.4 \pm 2.0$ & $7.0 \pm 1.1$ & 0.76418 \\
\hline Mean time to recurrence after initial op \pm SD (mos) & $3.5 \pm 1.3$ & $2.8 \pm 1.6$ & 0.22246 \\
\hline MR-indicated complete resection following reop $(n=12)$ & $10 / 12(83)$ & $2 / 12(17)$ & 0.0001 \\
\hline MR-indicated incomplete resection following reop $(n=10)$ & - & $10 / 10(100)$ & \\
\hline ECoG \& MRI concordant $(n=15)$ & $10 / 15(67)$ & $5 / 15(33)$ & 0.005 \\
\hline ECoG \& MRI not concordant $(n=7)$ & - & $7 / 7(100)$ & \\
\hline
\end{tabular}


(10 [83\%] of 12 patients with complete resection vs 0 [0\%] of 10 with incomplete resections; $p=0.0001$ ). None of the other preoperative variables (duration of seizures, age at first and second surgery, and time to recurrence) were statistically significant, although patients who achieved seizure freedom had longer average duration to seizure recurrence following first surgery.

\section{iMRI}

iMRI was used in 9 of the 22 reoperations in our cohort (Cases 14-22; Case 21 used iMRI on the third resection). iMRI indicated residual dysplasia in 4 cases, resulting in a reexamination of the resection cavity, and return to surgery in 3 cases. Use of 1.5-T intraoperative or 3-T postoperative MRI confirmed complete resection in all cases, and each of these patients has achieved complete seizure freedom (Engel Class I).

\section{Safety and Postoperative Morbidity}

One patient developed an intermittent CSF leak from the site of incision. Four patients developed new or worsening hemiparesis (all expected). One patient developed sensory deficits (expected). Two patients developed visual deficits (exophoria and hemianopsia; all expected). There were no deaths in our cohort.

\section{Discussion}

Our results suggest that reoperation is clinically beneficial in patients with medically intractable epilepsy due to FCD who continue to have seizures following a first surgery, with the benefits of further resection justifying the risks of further surgery. We emphasize the need for an attitude of persistence in surgical intervention to achieve favorable seizure outcome. Reoperation was particularly beneficial in patients with concordant ECoG and MRI findings. We found that the combined utility of intraoperative ECoG and iMRI allowed us to achieve high rates of complete resection of residual dysplasia and seizure freedom in these patients. Patients with discordant ECoG and MRI findings may ultimately benefit from more aggressive resection earlier in treatment to allow more time for functional plasticity and recovery.

Despite reports of reoperation ranging from $6 \%$ to $26 \%$ in epilepsy surgery cohorts, few studies exist in pediatric populations. ${ }^{4,8,11,15,16,23}$ Recently, Ramantani et al. reported on achieving $61 \%$ seizure freedom and $83 \%$ favorable seizure control (Engel Class I and II) in a pediatric cohort of 23 patients undergoing reoperation after failed first surgery, the majority of whom presented with medically intractable epilepsy secondary to cortical dysplasia. ${ }^{16} \mathrm{We}$ present similar findings that demonstrate the benefit and low risk of reoperation in pediatric patients who do not respond well to first surgery. This work builds upon the previous literature to help identify clinical and diagnostic criteria necessary in deciding if and to what extent further resections should be undertaken in selected patients.

We sought to delineate factors associated with successful reoperations. We found that an important predictor of seizure freedom following second surgery was concordance of the MRI-indicated dysplastic foci with intraop- erative ECoG. Holmes et al. were the first to demonstrate the importance of concordance in EEG and MRI findings in patients undergoing further reoperations for intractable epilepsy. ${ }^{9}$ Similarly, we found that patients with concordant localization achieved higher rates of complete seizure freedom than patients with discordant localization. Furthermore, of the patients with concordant localization who continued to have seizures, all went on to achieve significant improvement in seizure frequency (Engel Class II) after accounting for all operations.

Patients in our cohort who presented with ECoG findings discordant with MRI localization of FCD foci did not clinically benefit from a lesionectomy or long-term grid monitoring followed by lesionectomy. Furthermore, in 3 of these cases (Cases 7, 9, and 13) the patient's seizure frequency increased from presurgical levels following repeat surgeries. It is therefore important to delineate criteria that may indicate a patient is not suitable for conservative focal lesionectomy, so that ultimately a more aggressive treatment can be undertaken at a younger age allowing the patient to achieve both seizure freedom and functional recovery.

Reoperation has been demonstrated to be clinically beneficial in patients with large epileptic zones who did not achieve desired seizure outcomes from first surgery due to limited lesionectomy. ${ }^{2,16}$ In these cases more radical operations (multilobar or hemispheric reoperations) lead to increased levels of complete resection and thus seizure freedom in pediatric patients with continued intractable epilepsy following first surgery. Of the 3 patients in our cohort with discordant ECoG and MRI findings who initially presented with large multilobar FCD, each ultimately benefited from aggressive multilobar resections: the patients in Cases 5 and 10 ultimately benefited from hemispherectomy on the third operation, and the patient in Case 3 ultimately benefitted from a multilobe lobectomy on the fourth operation. Importantly, postoperative histopathology suggested diffuse hemispheric dysplasia in each case. Hence, our results support the notion that in cases of suspected diffuse hemispheric FCD lesions leading to discordant preoperative evaluation, it may be clinically beneficial to offer more radical resections at early stages of treatment, thus allowing more time for neuroplastic compensation.

Extended repeat resections of residual focal lesions after unsuccessful first surgery have been shown to be clinically beneficial. ${ }^{20,26} \mathrm{In}$ accordance with these findings, we found that patients with MRI-indicated focal lesions concordant with ECoG localization-who had failed first surgery because either the surgical goal was not carried out completely as planned or due to functional considerations-achieved the best outcomes after reoperation in our cohort. We found that in such cases, the combined utility of ECoG (both subdural ECoG for surface FCD and depth electrodes for deeper lesions) and neuronavigation were imperative for localization of residual dysplasia at the beginning of the operation; however, intraoperatively, we found guidance with iMRI to be most useful in localizing and confirming a complete resection had been accomplished.

Previous studies ${ }^{12,17,18,21,22}$ have illustrated the utility 
of iMRI as an intraoperative quality control mechanism that leads to high rates of complete resection and reduces the need for reoperation. In this study we report on the further utility of iMRI in reoperations in which the first (non-iMRI) surgery did not achieve surgical goals due to the inability to intraoperatively detect residual dysplasia at the margins. This utility became most evident in patients with insular or peri-eloquent lesions. Due to the combined subtle nature of FCD lesions, difficulty in compensating for intraoperative brain shifts, and concern for functional deficits, these cases pose increased difficulty in lesional resections. We note that in one-third of the cases utilizing iMRI in our cohort, intraoperative detection of residual dysplasia resulted in modification of the surgical plan of action and extension of the resection. Overall, we achieved complete resection in all cases utilizing iMRI.

We found that an important predictor of seizure freedom was localization and complete resection of the MRIlocalized FCD foci. There exists, however, a number of possible limitations to MRI localization of FCD. In addition to cases of MRI-negative cortical dysplasia or diffuse hemispheric FCD in which MRI would not be beneficial, Case 3 presents a notable possible limitation particularly relevant to reoperation cases. The neuroradiologist reported a complete resection with abnormal T2 signal related to gliosis along the posterior inferior margin of the resection cavity following reoperation. Postoperative histopathological analysis, however, confirmed residual FCD at the margins of the cavity that were likely skewed by the gliosis on MRI. FCD lesions are typically subtle and difficult to differentiate from surrounding cortex. This difficulty can be compounded by the presence of abnormal myelination, gliotic scaring, and white matter abnormalities, which can appear larger than the FCD lesion. ${ }^{25}$ Future imaging studies may seek to address the intraoperative resolution of the FCD lesions in this context.

\section{Limitations of the Study}

The limitations of our study are inherent to its retrospective nature. Determination of whether ECoG was concordant with the MRI-indicated FCD foci was derived largely from the surgical record, which may have skewed our analysis. Because this study spans several neurosurgeons and clinical neurophysiologists, there is the possibility of some degree of operator dependency bias. While our median follow-up period is well over a year in duration, some patients have relatively short follow-up durations that may skew data toward those doing well following reoperation. Furthermore, our small sample size precludes the ability to provide definitive support of prognostic factors or tools that improve clinical outcomes.

\section{Conclusions}

We found reoperations to be beneficial in the majority of patients in our cohort, with more than half $(n=13)$ achieving complete seizure freedom. Patients with concordant $\mathrm{iECoG}$ and MRI localization benefit from extended resection of MRI-indicated residual dysplasia, and iMRI may be helpful as a quality control mechanism to ensure that a complete resection has been accomplished. Patients with discordant findings may benefit from more aggressive resections at earlier stages to achieve better seizure control and ensure functional plasticity.

\section{Acknowledgments}

We wish to acknowledge Xin He, BS, and Valeiry Korostysheviskiy, $\mathrm{PhD}$, for their assistance with statistical analysis. This study was supported in part by an Alpha Omega Alpha Carolyn L. Kuckein Student Research Fellowship.

\section{References}

1. Alexandre V Jr, Walz R, Bianchin MM, Velasco TR, TerraBustamante VC, Wichert-Ana L, et al: Seizure outcome after surgery for epilepsy due to focal cortical dysplastic lesions. Seizure 15:420-427, 2006

2. Bower RS, Wirrell EC, Eckel LJ, Wong-Kisiel LC, Nickels $\mathrm{KC}$, Wetjen NM: Repeat resective surgery in complex pediatric refractory epilepsy: lessons learned. J Neurosurg Pediatr 16:94-100, 2015

3. Cohen-Gadol AA, Ozduman K, Bronen RA, Kim JH, Spencer DD: Long-term outcome after epilepsy surgery for focal cortical dysplasia. J Neurosurg 101:55-65, 2004

4. Dunkley C, Kung J, Scott RC, Nicolaides P, Neville B, Aylett SE, et al: Epilepsy surgery in children under 3 years. Epilepsy Res 93:96-106, 2011

5. Engel J, Van Ness PC, Rasmussen TB, Ojemann LM: Outcome with respect to epileptic seizures, in Engel $\mathrm{J} \mathrm{Jr}$ (ed): Surgical Treatment of the Epilepsies, ed 2. New York: Raven Press, 1993, pp 609-621

6. Fauser S, Essang C, Altenmüller DM, Staack AM, Steinhoff BJ, Strobl K, et al: Long-term seizure outcome in 211 patients with focal cortical dysplasia. Epilepsia 56:66-76, 2015

7. Fountas KN, King DW, Meador KJ, Lee GP, Smith JR: Epilepsy in cortical dysplasia: factors affecting surgical outcome. Stereotact Funct Neurosurg 82:26-30, 2004

8. Hemb M, Velasco TR, Parnes MS, Wu JY, Lerner JT, Matsumoto JH, et al: Improved outcomes in pediatric epilepsy surgery: the UCLA experience, 1986-2008. Neurology 74:1768-1775, 2010

9. Holmes MD, Wilensky AJ, Ojemann LM, Ojemann GA: Predicting outcome following reoperation for medically intractable epilepsy. Seizure 8:103-106, 1999

10. Kim DW, Lee SK, Chu K, Park KI, Lee SY, Lee CH, et al: Predictors of surgical outcome and pathologic considerations in focal cortical dysplasia. Neurology 72:211-216, 2009

11. Krsek P, Maton B, Jayakar P, Dean P, Korman B, Rey G, et al: Incomplete resection of focal cortical dysplasia is the main predictor of poor postsurgical outcome. Neurology 72:217-223, 2009

12. Kuhnt D, Becker A, Ganslandt O, Bauer M, Buchfelder M, Nimsky C: Correlation of the extent of tumor volume resection and patient survival in surgery of glioblastoma multiforme with high-field intraoperative MRI guidance. Neuro Oncol 13:1339-1348, 2011

13. Oluigbo CO, Wang J, Whitehead MT, Magge S, Myseros JS, Yaun A, et al: The influence of lesion volume, perilesion resection volume, and completeness of resection on seizure outcome after resective epilepsy surgery for cortical dysplasia in children. J Neurosurg Pediatr 15:644-650, 2015

14. Park CK, Kim SK, Wang KC, Hwang YS, Kim KJ, Chae JH, et al: Surgical outcome and prognostic factors of pediatric epilepsy caused by cortical dysplasia. Childs Nerv Syst 22:586-592, 2006

15. Ramantani G, Kadish NE, Strobl K, Brandt A, Stathi A, Mayer H, et al: Seizure and cognitive outcomes of epilepsy surgery in infancy and early childhood. Eur J Paediatr Neurol 17:498-506, 2013 
16. Ramantani G, Strobl K, Stathi A, Brandt A, Schubert-Bast $\mathrm{S}$, Wiegand G, et al: Reoperation for refractory epilepsy in childhood: a second chance for selected patients. Neurosurgery 73:695-704, 2013

17. Sacino MF, Ho CY, Murnick J, Tsuchida T, Magge SN, Keating RF, et al: Intraoperative MRI-guided resection of focal cortical dysplasia in pediatric patients: technique and outcomes. J Neurosurg Pediatr 17:672-678, 2016

18. Senft C, Bink A, Franz K, Vatter H, Gasser T, Seifert V: Intraoperative MRI guidance and extent of resection in glioma surgery: a randomised, controlled trial. Lancet Oncol 12:997-1003, 2011

19. Shah MN, Leonard JR, Inder G, Gao F, Geske M, Haydon $\mathrm{DH}$, et al: Intraoperative magnetic resonance imaging to reduce the rate of early reoperation for lesion resection in pediatric neurosurgery. J Neurosurg Pediatr 9:259-264, 2012

20. Shaver EG, Harvey AS, Morrison G, Prats A, Jayakar P, Dean P, et al: Results and complications after reoperation for failed epilepsy surgery in children. Pediatr Neurosurg 27:194-202, 1997

21. Siegel AM, Cascino GD, Meyer FB, Marsh WR, Scheithauer BW, Sharbrough FW: Surgical outcome and predictive factors in adult patients with intractable epilepsy and focal cortical dysplasia. Acta Neurol Scand 113:65-71, 2006

22. Sommer B, Grummich P, Coras R, Kasper BS, Blumcke I, Hamer HM, et al: Integration of functional neuronavigation and intraoperative MRI in surgery for drug-resistant extratemporal epilepsy close to eloquent brain areas. Neurosurg Focus 34(4):E4, 2013

23. Steinbok P, Gan PY, Connolly MB, Carmant L, Barry Sinclair D, Rutka J, et al: Epilepsy surgery in the first 3 years of life: a Canadian survey. Epilepsia 50:1442-1449, 2009

24. Wagner J, Urbach H, Niehusmann P, von Lehe M, Elger CE,
Wellmer J: Focal cortical dysplasia type IIb: completeness of cortical, not subcortical, resection is necessary for seizure freedom. Epilepsia 52:1418-1424, 2011

25. Widjaja E, Zarei Mahmoodabadi S, Otsubo H, Snead OC, Holowka S, Bells S, et al: Subcortical alterations in tissue microstructure adjacent to focal cortical dysplasia: detection at diffusion-tensor MR imaging by using magnetoencephalographic dipole cluster localization. Radiology 251:206-215, 2009

26. Wyler AR, Hermann BP, Richey ET: Results of reoperation for failed epilepsy surgery. J Neurosurg 71:815-819, 1989

\section{Disclosures}

The authors report no conflict of interest concerning the materials or methods used in this study or the findings specified in this paper.

\section{Author Contributions}

Conception and design: Oluigbo, Sacino. Acquisition of data: Oluigbo, Sacino. Analysis and interpretation of data: Oluigbo, Sacino. Drafting the article: Oluigbo, Sacino. Critically revising the article: Oluigbo, Sacino, Whitehead, Gaillard. Reviewed submitted version of manuscript: Oluigbo, Sacino, Ho, Kao, DepositarioCabacar, Myseros, Magge, Keating, Gaillard. Study supervision: Oluigbo, Gaillard.

\section{Correspondence}

Chima O. Oluigbo, Department of Neurosurgery, Children's National Medical Center, 111 Michigan Ave. NW, Washington, DC 20010. email: coluigbo@cnmc.org. 